\title{
A New Half-blind Algorithm of Smart Antenna for Mobile Terminal
}

\author{
Yuan $\mathrm{Li}^{\mathrm{a}}$, Wei Zhang ${ }^{\mathrm{b}}$, Jia Guo ${ }^{\mathrm{c}}$
}

Institute of Electronic and Information Engineering, Tianjin University, Tianjin, China, 300072

\begin{abstract}
The core of smart antenna is its adaptive algorithm. For the characteristics of TD-SCDMA mobile terminal, we analyze the advantages and disadvantages of various algorithms and propose a semi-blind algorithm combining the NLMS algorithm and the CMA algorithm in this paper. The new semi-blind algorithm has strong robustness and low complexity. It is suitable for smart antenna of TD-SCDMA mobile terminals.
\end{abstract}

Index Terms: TD-SCDMA mobile terminals; smart antenna; semi-blind algorithm

(C) 2011 Published by MECS Publisher. Selection and/or peer review under responsibility of the Research Association of Modern Education and Computer Science

Since the limitation of size, power consumption cost and other factors of smart antenna, the complexity of algorithm should be low, simple and fast convergence by the general requirements. So selecting an appropriate algorithm is a key issue. The environment of the mobile terminal is different from that of the base station. Because of the characteristics of the movement of the mobile station, the source DOA and the channel characteristics are time-varying. Angular spread is large. Multipath is rich. Channel environment is poor. Algorithm based on space doas is suitable for the case of small angular spread. So it is not suitable for the mobile terminals. Algorithm based on the signal structure (such as CMA) can simply capture the strongest input constant envelope signal, and this signal may be interference signal, so it is not suitable for use in mobile terminals. Algorithm based on training sequence need not determine signal DOA and has fast convergence, strong robustness. It can still work well under the condition of changing continuously of the channel characteristics and also work well when the number of array elements is less than the number of sources. Therefore, the algorithm based on training sequence for smart antenna of mobile terminal is the preferred algorithm. In CDMA system, uplink and downlink are assigned to each user specific or auxiliary pilot channels to send guidance signals in order to carry on synchronization and power control functions. Therefore, it is feasible for the mobile terminal to use the algorithm based on training sequence. The NLMS algorithm and the RLS algorithm are two widely used algorithms based on training sequence. Although the convergence rate of RLS algorithm is much faster than the NLMS algorithm, the complexity of RLS algorithm is too high. From the actual situation of the mobile terminal, NLMS algorithm is a better choice. Of course, the researchers invented a lot of the superior performance of algorithms, Such as Least Square Despread Respread Multitarget Array algorithm, LS-DRMTCMA, OVS-LMS, etc. These algorithms are based on the cost of increased complexity in exchange for improved system performance, suitable for base station.

* Corresponding author.

E-mail address: ${ }^{\text {liyuan@ @tju.edu.cn; }}$ b,c shanxi0403@163.com 
Convergence effect of algorithm based on training sequence depends on the reliability of the reference signal. If the correlation of reference signal and actual signal is better, the better the treatment effect. In the TD-SCDMA system, the time delay between the signals directly affect the relevance between the signals, the smaller the delay spread, the higher the degree of correlation signal. Therefore, the algorithm based on training sequence requires a system with precise synchronization and ideal channel estimation. The constant modulus algorithm, such as the CMA algorithm, generates a reference signal only according to the signal's constant amplitude characteristics, avoiding the limitations of the signal correlation. Experiments show that in land mobile communication system of high-speed transmission, using CMA adaptive array to get a good bit error rate performance. Therefore, the training sequence algorithm combined with the constant modulus algorithm will further enhance the robustness of the algorithm.

In TD-SCDMA system, each time slot has sudden 144chips training sequence. Under normal circumstances, NLMS algorithm in 144 iterations can achieve better convergence effect. However, in some harsh environments, the algorithm convergence in the training sequence cycle is not ideal, and can not be well in suppression of interference. At this time we should consider how to process the further error convergence after the training sequence. Reference [6] proposes a repeat iterative algorithm for the training sequence, but requires a higher update rate and memory space. TD-SCDMA system typically uses QPSK signal modulation, and its signal has the constant model characteristics. So we can use the combination of the training sequence-based algorithm and constant modulus algorithm to improve system performance. This proposes a semi-blind algorithm that is a combination of CMA algorithm and NLMS algorithm applied to the mobile terminal. We use NLMS algorithm in the 144chips' training cycle. If it can't reach the convergence effect in the training sequence cycle, we then use constant modulus algorithm in the signal cycle. The NLMS algorithm and compare the CMA algorithm can find the main difference is the error function for the NLMS algorithm

Comparing the NLMS algorithm with the CMA algorithm, we can find the main difference is that the error function of the NLMS algorithm is

$$
e(n)=d(n)-y(n)
$$

And the CMA error function is

$$
e(n)=\frac{y(n)}{|y(n)|}-y(n)
$$

Therefore, we consider training sequence $d(n)$ as a reference signal in the 144chips cycle with the use of NLMS algorithm, detecting the size of the error signal ${ }^{e(n)}$ after the training cycle has completed. If the ${ }^{e(n)}$ last 10 samples are below a certain threshold value, the algorithm stops. If the samples are higher than threshold value, we will further use the constant modulus characteristic sequence $\frac{y(n)}{|y(n)|}$ as a reference signal for CMA algorithm until the $e(n) 10$ consecutive samples reach under the threshold value. Because processing core of the NLMS algorithm and the CMA algorithm is the same (based on minimum mean square error criterion), switching between the algorithms need change the desired signal, so the whole process is very easy to implement by using hardware. Because CMA algorithm step factor for the input is fixed at the same time, we do not need to normalize each iteration so its implementation is simpler than the NLMS algorithm. For the choice of error threshold, the steady-state error for the NLMS algorithm is smaller than the CMA algorithm, so the error threshold value generally takes more than the value of steady-state error of CMA algorithm. Since this method has been adopted in the training cycle through the NLMS algorithm to achieve some suppression for the interference signal, it solves the follow-up disturbance capture question in the CMA algorithm, so that the CMA algorithm only converges to the desired signal. 
Semi-blind algorithm flow chart shows in Fig.1. We carry on the MATLAB simulation analysis of the semi-blind algorithm in case that the following sequence in the training cycle for the NLMS algorithm can't reach the threshold value. Parameters: interference signal direction: -30 degrees, the direction of the desired signal: 30 degrees, SIR:-5db, SNR: 20db, the length of stride factor: 0.05, the threshold squared error: 0.01; NLMS algorithm simulation results show in Fig.2, Fig.3. Obviously in the 144chips' training cycle, the NLMS algorithm has not reached the threshold value. Therefore, semi-blind algorithm iterates 144 NLMS, and then CMA iterative process begins in order to further reduce the error. Finally the directional diagram will make the main wave beam to aim at the desired signal, and simultaneously suppresses the disturbance to - $45 \mathrm{db}$. The simulation results show in Fig.4, Fig.5. Clearly, the semi-blind algorithm is superior to NLMS algorithm in the interference suppression depth and the desired signal direction gain. When time delay expands in a large way and the clock synchronization presents the deviation, the semi-blind algorithm can also work well.

Comparing with the NLMS algorithm, semi-blind algorithm strengthens environmental variation's adapt capacity (robustness), so it has some practical value.

From the algorithm flowchart, semi-blind algorithm only adds an error size module and the module of constant receiving signal amplitude in the NLMS algorithm foundation. The auto-adapted iterative core is invariable, so the hardware implementation complexity relative to the NLMS algorithm changes little. Because the steady-state error of the NLMS algorithm is less than CMA algorithm, the error after 144 iterations is lower than the error threshold value in many cases. Therefore we may also choose the entire process to determine the judgment of error. In the way of judging the whole process, it may reach the error threshold value with several iterations in better channel environmental conditions. The algorithm stops at this time. The advantage is that it needs fewer iteration times and saves power consumption, and the disadvantage is that the error will be slightly lager than that after completion of training cycle. 


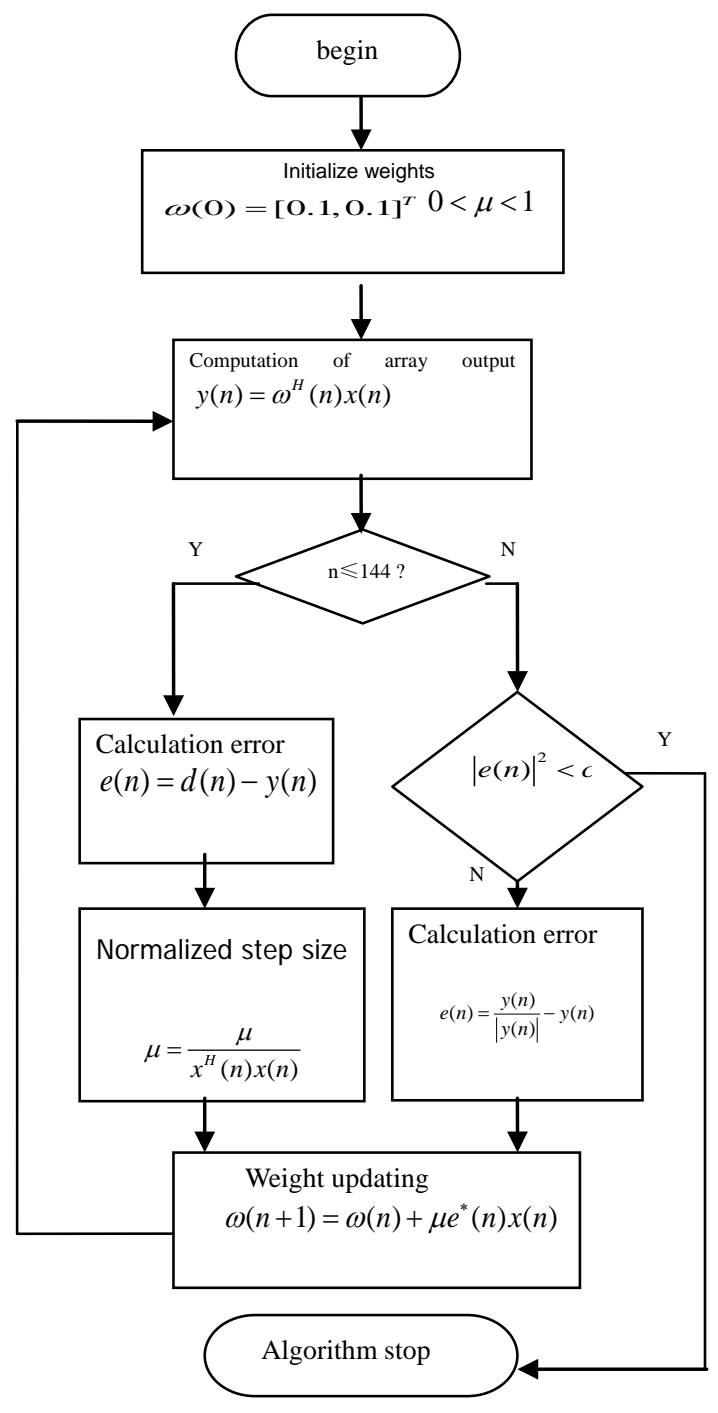

Fig.1 semi-blind Algorithm flowchart 


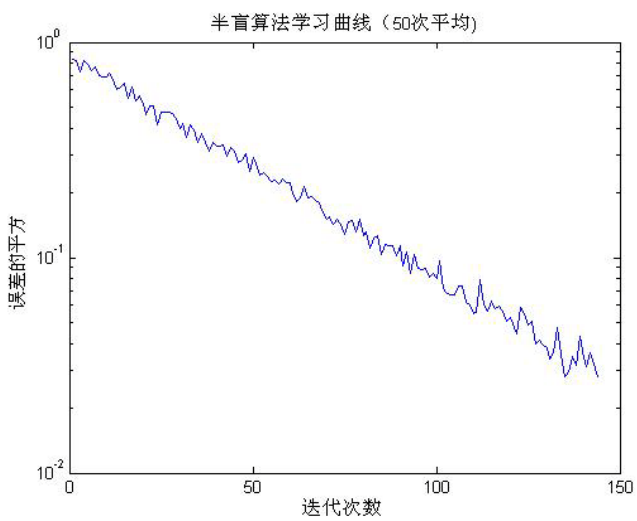

Fig.2 NLMS algorithm learning curve

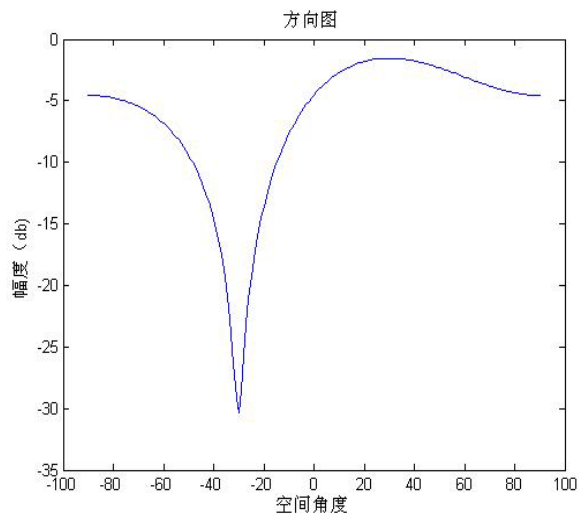

Fig.3 NLMS algorithm orientation image

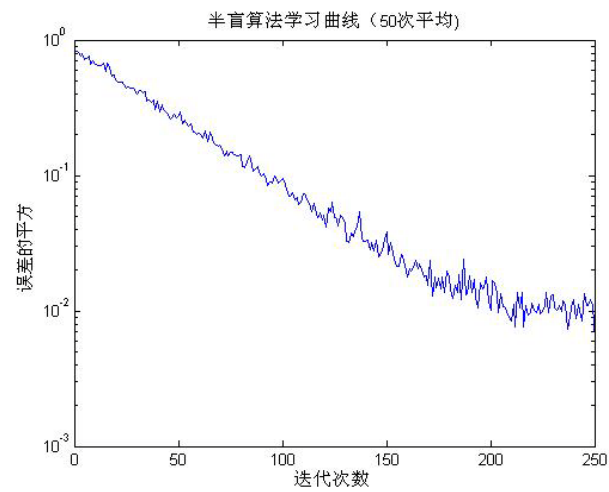

Fig.4 semi-blind algorithm learning curve 


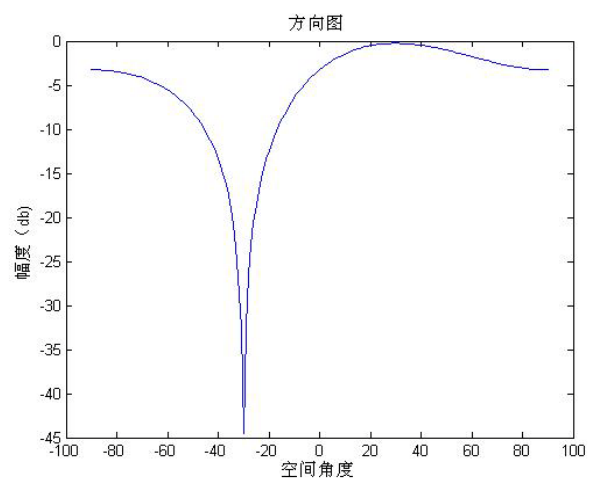

Fig.5 semi-blind algorithm orientation image

Conclusion: This article proposes a semi-blind algorithm that is combination of the NLMS algorithm and the CMA algorithm and it has great advantages compared to the NLMS algorithm. It solves the original algorithm slow convergence that can't reach error threshold value issue in the training sequence cycle. The algorithm has strong environmental adaptability. Simultaneously the complexity corresponds to NLMS algorithm, so it has good practical value. The new semi-blind algorithm has very strong toughness and low complexity. It is suitable for smart antenna of TD-SCDMA mobile terminals.

\section{Acknowledgment}

This work is sponsored by the National Basic Program of China (973 Program) (No.2007CB310605).

\section{References}

[1]YaoHuan Gong, Adaptive filter (Second Edition).—— Time-domain adaptive filtering and smart antennas, Beijing: Electronic Industry Press, 2003 (in Chinese)

[2]ZeMing Xie, ShenLi Lai, repeated iterative adaptive algorithm of TD-SCDMA system smart antenna, telecommunications technology, 2005, 4: 5-8 (in Chinese)

[3]Celik N, Kim W, Demirkol M F, etal, Implementation and Experimental Verification of Hybrid Smart-Antenna Beamforming Algorithm, IEEE Antennas and Wireless Propagation Letters, 2006, 5(1):280-283 [4]Huang F, Sheng W X, Qi B Y, etal, A new fast adaptive beamforming method based on LMS algorithm, APMC 2005, 2005, 3:4-7

[5]Daehyun Paik, Golden R M, Torlak M, etal, Blind adaptive CDMA processing for smart antennas using the block shanno constant modulus algorithm, IEEE Transaction on Signal Processing, 2006, 54(5):1956-1959

[6]Tingting An, New Wireless Communication System Research and Design of Antenna,2010,14-16 (in Chinese)

[7]Yinlan Zhu, Smart antenna used in modern mobile communication, Communication Technology,2010, (in Chinese)

[8]Jian Chen, Pen He, Yan Qin, Td-scdma smart antenna of test automation, Telecom Network Technology,2010,64-66 (in Chinese) 